\title{
Colonic gallstone ileus treated with radiologically guided extracorporeal shock wave lithotripsy followed by endoscopic extraction
}
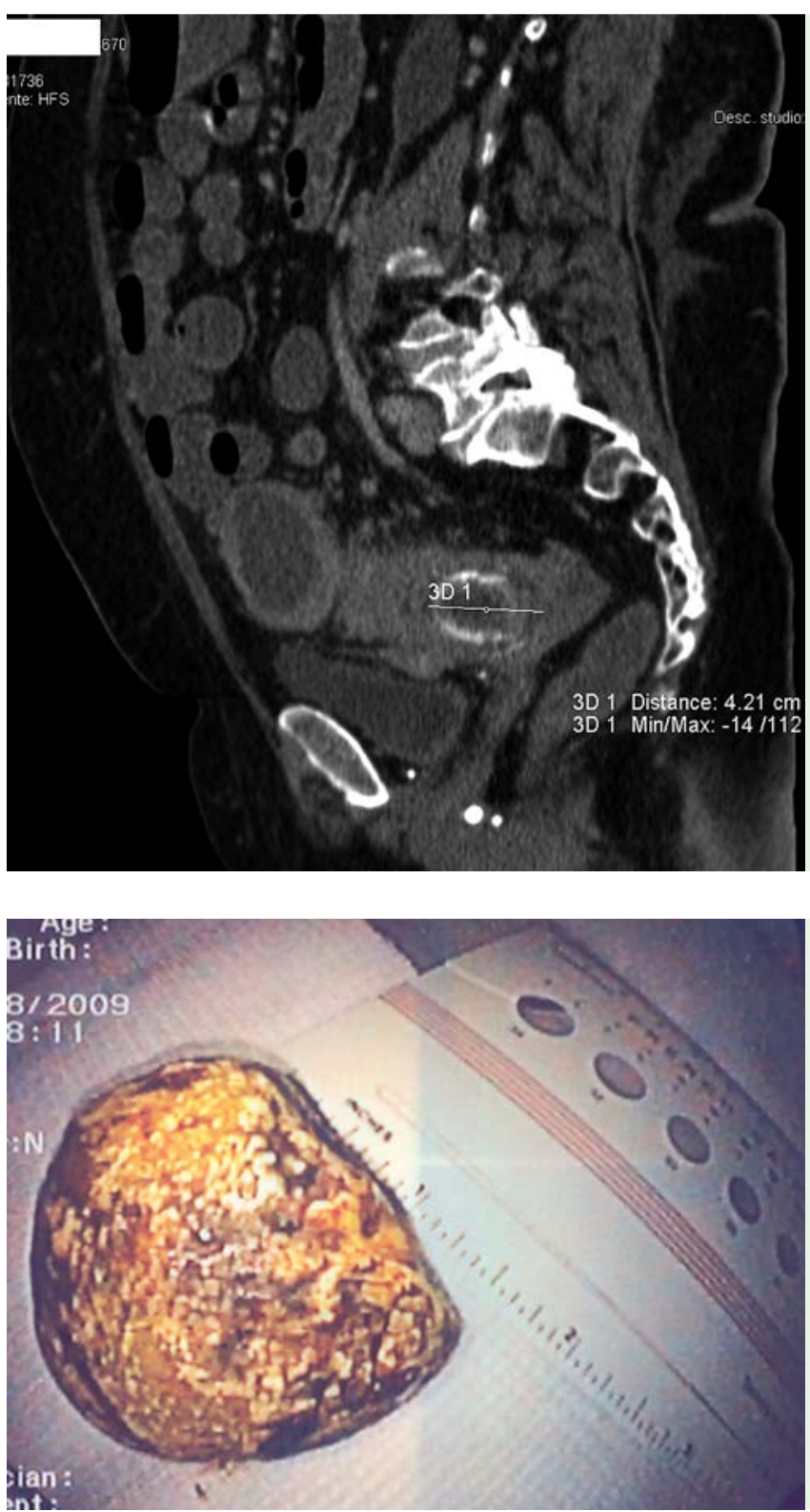

Gallstone ileus is a rare cause of mechanical bowel obstruction due to the passage of a solitary large gallstone into the gastrointestinal tract through a cholecystoenteric fistula [1]. Early surgical intervention is the treatment of choice; however, the surgical mortality rate is high as most patients are elderly and many suffer from concomitant diseases [2]. Ultrasound-guided extracorporeal shock wave lithotripsy (ESWL) has been suggested as an effective, noninvasive therapeutic al-
Fig. 1 Contrast-enhanced computed tomography (CT) scan showing air-fluid levels and a partially calcified stone at the rectosigmoid junction.

Fig. 3 The largest gallstone fragment, which was extracted endoscopically after extracorporeal shock wave lithotripsy (ESWL).

ternative to surgery. Cases of jejunal and gastric gallstone ileus treated with ESWL have already been described [3-5]. However, the use of ultrasound-guided ESWL is limited in the colon because the stones are often not radiopaque or only partially calcified, and are frequently covered by intestinal gas, which prevents clear localization of the gallstone. We describe a case of gallstone ileus of the colon that was successfully treated with radiologically guided ESWL that visualized the obstruc-

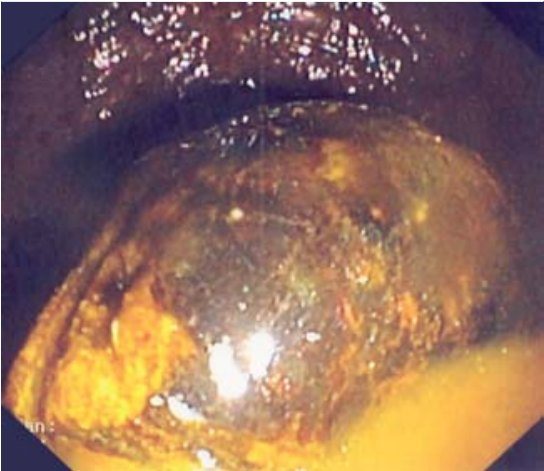

Fig.2 Endoscopic appearance of the obstructing gallstone in the sigmoid colon.

tion by focusing on a metallic clip that had been inserted during a previous rectosigmoidoscopy.

An 80-year-old woman was admitted to hospital with fever, constipation, and abdominal pain. A computed tomography (CT)-scan showed colonic distension, airfluid levels, and a 5-cm, partially calcified stone that was obstructing the colon at the rectosigmoid junction ( $\bullet$ Fig. 1 ). In addition, there was evidence of pneumobilia in the common bile duct and intrahepatic biliary ducts, and of a fistula from the gallbladder into the colon at the hepatic flexure. Ultrasonography failed to visualize the gallstone because of interference from abundant colonic gas, while rectosigmoidoscopy confirmed the presence of a large, nonremovable biliary stone ( Fig. 2). During the endoscopy, a metallic clip was inserted into the colonic mucosa close to the obstruction so that the area to be treated could be visualized radiologically. The patient subsequently underwent three sessions of ESWL focusing radiologically on the metallic clip inserted adjacent to the obstruction. The treatments were well tolerated, with no analgesia or sedation required, and allowed the gallstone to be successfully fragmented and removed during a further rectosigmoidoscopy ( Fig.3). A subsequent Gastrografin enema confirmed the presence of a cholecystocolic fistula and resolution of the colonic obstruction after removal of the gallstone ( Fig.4). 


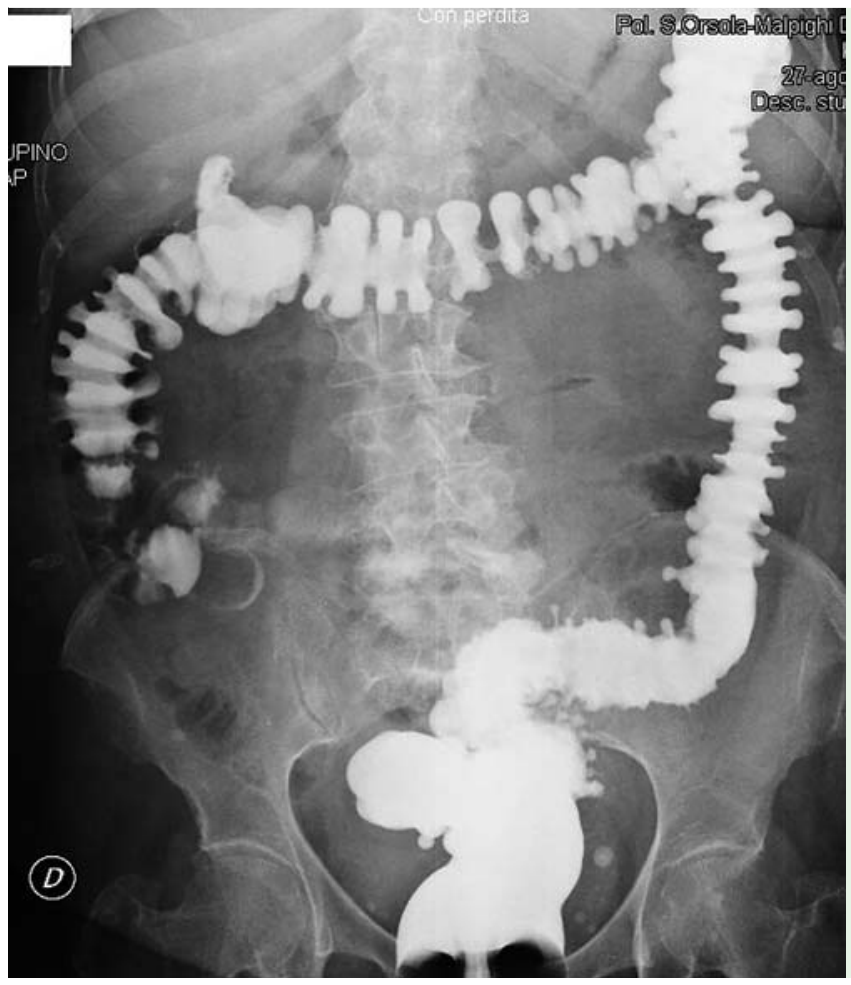

Fig. 4 Gastrografin enema showing the cholecystocolic fistula and resolution of the colonic obstruction after removal of the gallstone.

Endoscopy_UCTN_Code_CCL_1AD_2AJ

Competing interests: None

\section{R. Muratori, V. Cennamo, M. Menna, P. Cecinato, L. H. Eusebi, G. Mazzella, F. Bazzoli}

Department of Internal Medicine and Gastroenterology, University of Bologna, Policlinico S.Orsola-Malpighi, Bologna, Italy

\section{References}

1 Beuran M, Ivanov I, Venter MD. Gallstone ileus - clinical and therapeutic aspects. J Med Life 2010; 3: 365-371

2 Moberg AC, Montgomery A. Laparoscopically assisted or open enterolithotomy for gallstone ileus. Br J Surg 2007; 94: 53-57

3 Meyenberger C, Michel C, Metzger $U$ et al Gallstone ileus treated by extracorporeal shockwave lithotripsy. Gastrointest Endosc 1996; 43: 508-511

4 Murray EL, Collie M, Hamer-Hodges DW. Colonoscopic treatment of gallstone ileus. Endoscopy 2006; 38: 197

5 Dumonceau JM, Delhaye M, Cremer M. Extracorporeal shock-wave lithotripsy for gallstone ileus. Gastrointest Endosc 1996; 44: 759

\section{Bibliography}

DOI http://dx.doi.org/

10.1055/s-0031-1291641

Endoscopy 2012; 44: E88-E89

(c) Georg Thieme Verlag KG

Stuttgart · New York

ISSN 0013-726X

\section{Corresponding author}

\section{R. Muratori, MD}

Dipartimento di Medicina Interna e

Gastroenterologia

Università di Bologna

Policlinico S.Orsola Malpighi Via Massarenti 9

40138 Bologna

Italy

Fax: +39-051-6364112

rosangela.muratori@aosp.bo.it 